ARTICLE

\title{
Enhancing sub-bandgap external quantum efficiency by photomultiplication for narrowband organic near-infrared photodetectors
}

\author{
Jonas Kublitski (i) ${ }^{1 \times}$, Axel Fischer ${ }^{1}$, Shen Xing ${ }^{1}$, Lukasz Baisinger (iD) ${ }^{1}$, Eva Bittrich (iD ${ }^{2}$, Donato Spoltore ${ }^{1}$, \\ Johannes Benduhn (10) ${ }^{1}$, Koen Vandewal ${ }^{3}$ \& Karl Leo ${ }^{1,4 凶}$
}

Detection of electromagnetic signals for applications such as health, product quality monitoring or astronomy requires highly responsive and wavelength selective devices. Photomultiplication-type organic photodetectors have been shown to achieve high quantum efficiencies mainly in the visible range. Much less research has been focused on realizing near-infrared narrowband devices. Here, we demonstrate fully vacuum-processed narrowand broadband photomultiplication-type organic photodetectors. Devices are based on enhanced hole injection leading to a maximum external quantum efficiency of almost $2000 \%$ at $-10 \mathrm{~V}$ for the broadband device. The photomultiplicative effect is also observed in the charge-transfer state absorption region. By making use of an optical cavity device architecture, we enhance the charge-transfer response and demonstrate a wavelength tunable narrowband photomultiplication-type organic photodetector with external quantum efficiencies superior to those of pin-devices. The presented concept can further improve the performance of photodetectors based on the absorption of charge-transfer states, which were so far limited by the low external quantum efficiency provided by these devices.

\footnotetext{
${ }^{1}$ Dresden Integrated Center for Applied Physics and Photonic Materials (IAPP) and Institute for Applied Physics, Technische Universität Dresden, Dresden, Germany. ${ }^{2}$ Leibniz-Institut für Polymerforschung Dresden e.V., Dresden, Germany. ${ }^{3}$ Instituut voor Materiaalonderzoek (IMO-IMOMEC), Hasselt University, Diepenbeek, Belgium. ${ }^{4}$ Center for Advancing Electronics Dresden (cfaed), Technische Universität Dresden, Dresden, Germany. ${ }^{凶}$ email: jonas. kublitski@tu-dresden.de; karl.leo@tu-dresden.de
} 
F rom simple automatic lights in the halls of our buildings to the cruise control of cars, photodetectors (PDs) are playing a major role in everyday life ${ }^{1}$. Often, fast detection of faint signals is required, which is currently provided by inorganic avalanche photodiodes ${ }^{2}$. As the automotive industry is moving towards self-driving $\mathrm{cars}^{3}$, properties like lower cost, higher sensitivity, wavelength selectivity, and form-free devices are required $^{4,5}$. PDs made from organic semiconductors can offer these properties, but need further research to optimize these devices for low intensity signals, i.e., to reach high specific detectivities ${ }^{6}$.

Photomultiplication-type organic photodetectors (PM-OPDs) are capable of amplifying small photocurrents without requiring external/additional circuit components. This can be achieved by a photo-induced enhanced injection via energy level bending caused by charge accumulation near the injecting electrode ${ }^{7,8}$. Following its observation in single material active layers ${ }^{9}$ and donor-acceptor (D-A) heterojunctions ${ }^{10}$, different strategies have been introduced to achieve such charge accumulation and thereby the required energy level bending through a lack of percolation path for one charge carrier type $\mathrm{e}^{11-14}$, energetic barriers via interfacial layers ${ }^{15,16}$ as well as intentionally inserted trap states $^{17,18}$. All of these strategies include an accumulation of one carrier type near the contact, such that the electrical field caused by these charges bends the energy level, enabling the opposite charge to be injected via tunneling across the injection barrier ${ }^{7}$. If the transit time of injected charge carrier is lower than the lifetime of the accumulated, photo-generated charges, an EQE > $100 \%$ is observed. Here, we would like to stress that prior to the photomultiplication process the photon needs to be absorbed by the active layer. We therefore conclude that the minimum criteria for photomultiplication is that the internal quantum efficiency (IQE) is larger than unity.

The effect described above has been applied in organic and hybrid PDs, leading to external quantum efficiencies (EQEs) as high as $10^{5} \%^{19-21}$. Nonetheless, the specific detectivity $\left(D^{*}\right)$ achieved by these devices, which takes into account not only EQE but also the device noise current, ranges from $10^{10}$ to $10^{15} \mathrm{~cm}$ $\mathrm{Hz}^{1 / 2} \mathrm{~W}^{-1}$ (Jones) in the visible range ${ }^{7}$, values comparable to those of diode-like OPDs. Guo et al. presented two different polymers blended with zinc-oxide nanoparticles, reaching $D^{*}$ of $10^{15}$ Jones in the ultraviolet region ${ }^{17}$. In the near-infrared (NIR) at $1200 \mathrm{~nm}$, using colloidal lead sulfide $(\mathrm{PbS})$ quantum dots, Lee et al. achieved $D^{*}$ of $10^{13}$ Jones $^{22}$, while $10^{14}$ Jones was attained for polymer-based devices in the visible range $\mathrm{e}^{23}$. Recently, imagers $^{24}$ and dual band ${ }^{25}$ OPDs were also fabricated using photomultiplication, with $D^{*}$ of $\sim 10^{14}$ and $\sim 10^{13}$ Jones, respectively. Moreover, photomultiplication has been also explored in perovskites $^{26}$, for which EQE of $4500 \%$ and $D^{*}$ of $10^{13}$ Jones were demonstrated at around $600 \mathrm{~nm}^{27}$. Despite the remarkable performance achieved by PM-OPDs in terms of increased EQE, limitations are still present in these class of devices. PM-OPDs suffer from high noise, a result of field dependent dark currents observed in these devices. In fact, this represents the main limitation in PM-OPDs as the gain acquired by biasing the device might also result in an increased dark current.

Photomultiplication has been extensively exploited in solutionprocessed organic/hybrid devices. However, despite the many advantages offered by sublimable small molecules, fewer examples were demonstrated in fully vacuum-processed devices ${ }^{10,15,28-30}$. Huang et al. demonstrated EQE higher than $1000 \%$ in devices based on $\mathrm{C}_{60}$. These values were attributed to the disordered structure of $\mathrm{C}_{60}$ and to interfacial traps at the interface $\mathrm{C}_{60} /$ hole transporting layer ${ }^{31}$. Similar results were achieved by interfacial blocking layers in hybrid (solution- and vacuum-processed) ${ }^{18,32}$ and fully vacuum-processed devices ${ }^{16,33}$, which are used to avoid charge extraction, thereby causing the necessary band bending. In general, the vacuum deposition provides the possibility of depositing a vertical gradient of donor or acceptor molecules in the blend, as well as fine tuning the mixing ratio. Yet, such fine tuning extensively used in solution-processed PM-OPDs has not been investigated in vacuum-processed devices. Besides that, vacuum deposition offers the possibility of sequentially stacking of multiple layers, the well-established doping technology 34,35 , straightforward fabrication of matrices of individual pixels, and is for commercial organic optoelectronic devices the currently preferred manufacturing technique.

Another aspect not considered in PM-OPDs concerns photomultiplication in the extended charge-transfer (CT) state absorption region. With the aid of a Fabry-Perot microcavity, this rather weak absorption region has been used in NIR narrowband organic photodetectors (CT-OPDs) ${ }^{36-38}$. Such narrowband OPDs could significantly benefit from the increased IQE, if photomultiplication would take place also by direct excitation of CT states. However, it is unclear whether direct excitation of CT states can result in a photomultiplication process. Utilizing the intermolecular CT state absorption renders possible to detect NIR photons beyond $1700 \mathrm{~nm}$ meanwhile using rather small and sublimable organic semiconductors where the absorption profile can be easily tuned by the D-A system. On the other hand, the weak intermolecular absorption cross section ${ }^{39,40}$ challenges the overall performance and here the photomultiplication could improve the electrical performance by increasing the gain for every absorbed photon.

Here, we report a fully vacuum-processed PM-OPD based on low-acceptor content (3 wt\%) $\mathrm{ZnPc}_{\mathrm{C}} \mathrm{C}_{60}$ material system, with a maximum EQE of almost $2000 \%$ achieved at $-10 \mathrm{~V}$. Additionally, an optimum operation regime maximizing EQE while keeping a low dark current is found, leading to a $D^{*}$ of $2.2 \times 10^{12}$ Jones at $670 \mathrm{~nm}$. Sensitively measured EQE spectra reveal that direct excitation of CT states also results in photomultiplication, which is confirmed by an IQE higher than $100 \%$ over the entire absorption region. Under $-5 \mathrm{~V}$ reverse bias, the $\mathrm{EQE}$ of the PMOPD surpasses that of an optimized pin-photodiode, demonstrating the potential for application in microcavity CT-OPDs. Indeed, by exchanging the transparent ITO contact with semitransparent Ag mirrors, while varying the thicknesses of the optical microcavity from 355 to $400 \mathrm{~nm}$, peaks in the NIR region originating from cavity enhanced CT absorption arise. Narrowband PM-OPDs show peak EQEs from 20 to $80 \%$ under $-10 \mathrm{~V}$ with full width at half maximum (FWHM) from 20 to $40 \mathrm{~nm}$, and $D^{*}$ of around $10^{11}$ Jones for all the resonant wavelengths. These results are comparable with narrowband organic pin-photodiodes based on cavities ${ }^{36,38}$, and higher than that of narrowband photomultiplication-type devices based on charge injection narrowing $(\mathrm{CIN})^{41}$. The concept presented here can be used to boost EQE of CT-OPDs, which so far was mainly limited by the low absorption cross section of CT states, the low internal quantum efficiency ${ }^{38}$, and the parasitic absorption of the contacts.

\section{Results}

Controlling the mixing ratio is essential for the working principle of previously reported PM-OPDs. For enhanced hole injection in reverse bias, electrons must accumulate near the cathode: we designed our devices based on a low-acceptor content (3 wt\%), such that few percolation paths are formed. The well-known $\mathrm{ZnPc}: \mathrm{C}_{60}$ system is chosen given the LUMO energy offset between these materials. At this concentration, electrons are intentionally trapped within the LUMO level of $\mathrm{C}_{60}$ and the bending caused by electron accumulation in the $\mathrm{C}_{60}$ phase leads to EQEs above $100 \%$. Below, we describe how this can be 
a

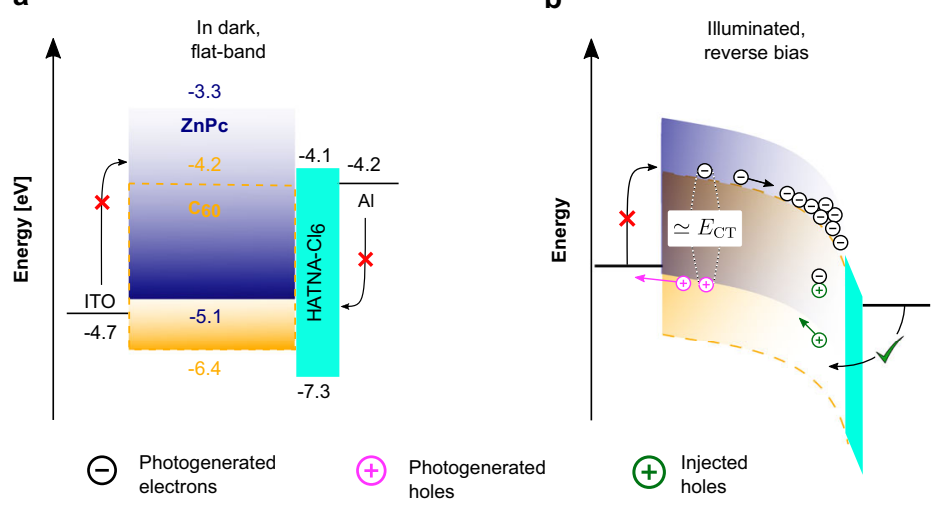

C

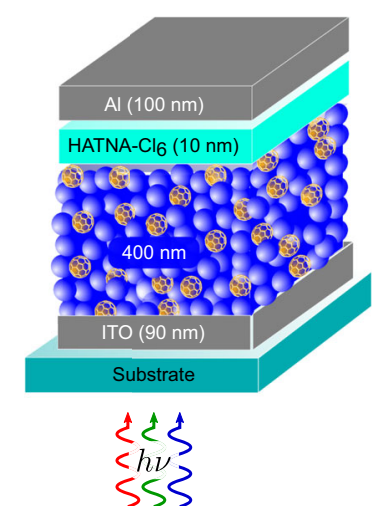

d

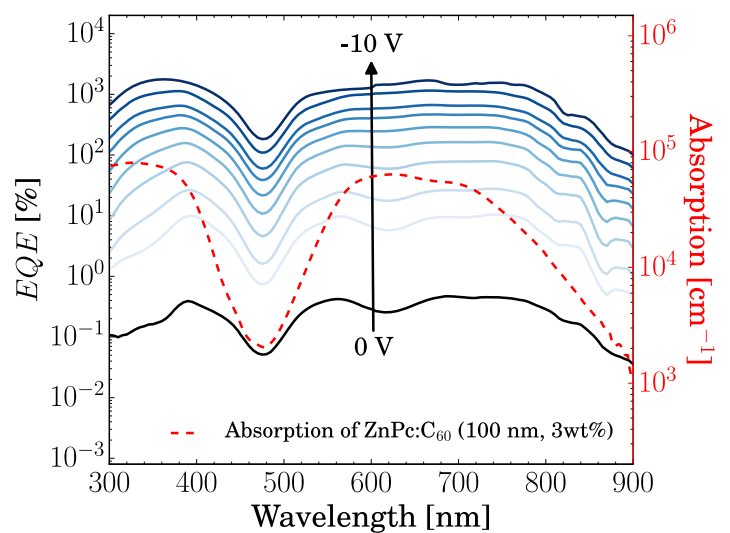

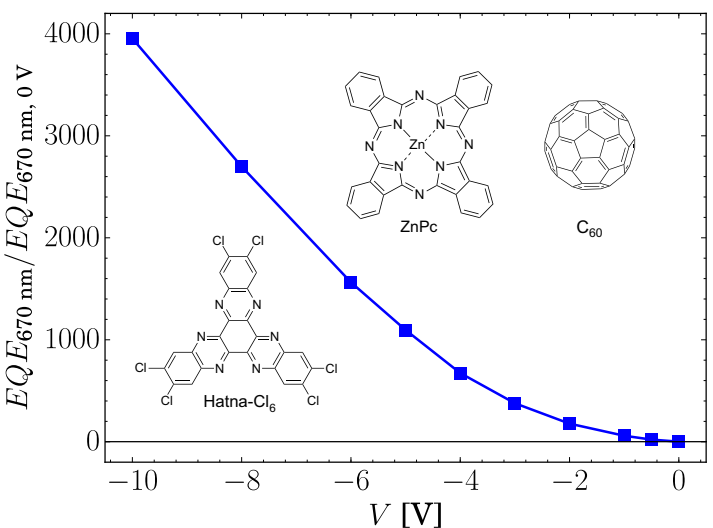

Fig. 1 Operation, device structure, and EQE of a PM-OPD. Schematic energy diagram a under dark at flat band condition and $\mathbf{b}$ negatively biased under illumination. c schematic device structure. d Voltage-dependent EQE (solid lines) of the device shown in c comprising ZnPc blended with $\mathrm{C}_{60}$ at 3 wt\%. Each line corresponds to one symbol in $\mathbf{d}$. Dashed red line shows the absorption spectrum of the same blend. Additionally, in $\mathbf{e}$, the relative enhancement factor as a function of applied reverse bias is presented. Symbols show the ratio between EQE at $670 \mathrm{~nm}$ at each voltage from $\mathbf{d}$ normalized by the EQE at $670 \mathrm{~nm}$ at $\mathrm{OV}$. The blue line is a guide to the eye. Note that no saturation is observed, indicating that EQE can be further increased. Inset shows the chemical structure of $\mathrm{C}_{60}, \mathrm{ZnPC}$ and $\mathrm{HATNA}-\mathrm{Cl}_{6}$. The energy level values of $\mathrm{ZnPC}_{2} \mathrm{C}_{60}$, and $\mathrm{HATNA} \mathrm{Cl}_{6}$ in a are taken from the literature ${ }^{55-57}$.

achieved in this system and how this effect can be used in narrowband PM-OPD.

Enhancing the external quantum efficiency. The PM-OPD operation in dark and under light as well as the architecture are shown in Fig. 1a-c. The bulk heterojunction comprising low $\mathrm{C}_{60}$ content ( $3 \mathrm{wt} \%)$ is sandwiched between two contacts, top Aluminum and bottom ITO, from which light enters the device, as shown in Fig. 1c. Pristine HATNA- $\mathrm{Cl}_{6}$ is used as hole blocking layer $(\mathrm{HBL})$ in between active layer and $\mathrm{Al}$ electrodes to reduce the reverse dark current. However, the thickness of HATNA-Cl 6 must be carefully controlled such that injection is enabled upon band bending. Under reverse bias in the dark, the high injection barriers (Fig. 1a) hinder holes and electrons to be injected into ZnPc and $\mathrm{C}_{60}$, respectively. Under forward bias, electrons and hole are injected into the device, such that the device behaves similarly to a photodiode. When the device is exposed to illumination, excitons are formed, and free charges are generated at $\mathrm{D}-\mathrm{A}$ interface. Due to absence of percolation paths, electrons are trapped in the acceptor phase and their transport is further hindered by the low electron mobility of the electrically undoped HATNA-Cl ${ }_{6}$ layer ${ }^{42}$. While $\mathrm{n}$-doped HATNA-Cl 6 has been already employed as an electron transport layer, in this device, we intentionally use a pristine layer such that the electron extraction is hindered and slowed down, which helps the electron accumulation at the cathode. This accumulation of electrons upon illumination causes that the energy levels bend in the vicinity of the contact, enabling holes from the external circuit to tunnel through the energy barrier imposed by the HATNA-Cl ${ }_{6}$ layer into the donor phase, where they are efficiently transported together with the photo-induced holes towards the anode.

From the process described above, a voltage-dependent increase in EQE is expected, as higher reverse voltage further decreases the energy barrier for injection. The black line in Fig. 1d shows the EQE measured at $0 \mathrm{~V}$, for which a maximum of $0.5 \%$ is achieved. This rather low value can be explained by the interrupted percolation path for electrons ${ }^{43}$ and charge separation probability at this concentration as well as by the unoptimized device architecture for $0 \mathrm{~V}$ operation. Clearly, no photomultiplication is observed. To prove the described working mechanism, we increase the reverse applied bias from 0 to $-10 \mathrm{~V}$. Indeed, the EQE rises accordingly, reaching almost $2000 \%$ at $-10 \mathrm{~V}$. Further increase is expected for higher negative bias, as can be seen from Fig. 1e, where no saturation in the relative enhancement is observed. However, as it will be discussed below, an optimum operation regime exists in the range of $-2.5 \mathrm{~V}$, where the highest $D^{*}$ is achieved.

In spite of the extensive work performed by different groups on PM device structures ${ }^{10,16}$, this effect has not yet been utilized to increase EQE in the spectral region of CT state absorption. In DA systems, interaction between donor and acceptor results in an extended but weak absorption band related to an optical transition from the HOMO of the donor to the LUMO of the 
acceptor. Recently, enhanced CT state absorption photodetectors (CT-OPDs) have been introduced ${ }^{36-38}$, which could benefit from high gain for absorbed photons provided by photomultiplication. However, it is not clear whether photomultiplicative gain can be achieved for photons that directly excite the CT states. Before investigating $\mathrm{PM}$ gain in the $\mathrm{CT}$ absorption band, we first determine the optimum $\mathrm{D}$-A concentration to achieve PM, as well as the relation with dark current. Below, we investigate these issues in $\mathrm{ZnPc}_{6} \mathrm{C}_{60}$ based devices.

Effect of acceptor concentration on the photomultiplication. In the previous section, we showed that EQE can be enhanced by three orders of magnitude by photomultiplication based on electron accumulation. In polymeric systems based on the same effect, it is well accepted that low concentration of one material type (D or A) is necessary for attaining photomultiplication, a condition which has not been investigated for small molecule based devices. Zhang et al. reported an efficient photovoltaic effect at around $5 \mathrm{wt} \%$ donor content, which suggests that at such concentrations, hole transport takes place efficiently ${ }^{44}$. The minimum acceptor concentration required for a $\mathrm{BHJ}$ to work as a D-A photodiode has not been established for low-acceptor content systems.

To investigate the concentration dependence, we fabricated devices comprising concentrations from 1 to $4 \mathrm{wt} \%$. The results are depicted in Fig. 2. Devices comprising $1 \mathrm{wt} \%$ and $2 \mathrm{wt} \%$ mixing ratios do not show any amplification and behave as an unoptimized photodiode. For these devices, EQE does not overcome $100 \%$ and is limited by the poor free charge carrier generation of the system, which explains the slightly higher EQE of the blend at $2 \mathrm{wt} \%$, where more exciton dissociation centers are available.

At $3 \mathrm{wt} \%$, the photocurrent increases almost one order of magnitude at a given reverse voltage. This abrupt enhancement is a result of a sufficient accumulation of charges at the contact, leading to an increased injection. At $4 \mathrm{wt} \%$, the device still shows amplification, but the performance of the device deteriorated, which we attribute to a more efficient extraction of electrons at this concentration. If the concentration is further increased, percolation paths are formed and efficient extraction of both charge carrier types takes place. The device will then behave as a typical organic photodiode. From these results, we infer that an optimum concentration exists (in our case, $3 \mathrm{wt} \%$ ), where sufficient charges are trapped to cause energy level bending while providing enough free charge carrier generation. At concentrations higher than that, the injection is reduced either because

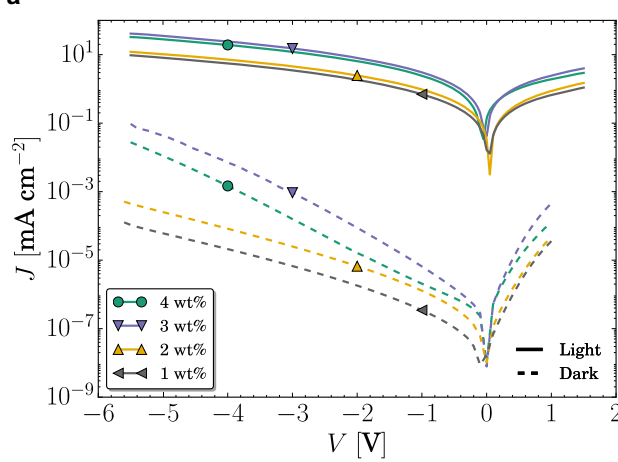

charges are extracted more efficiently or because the bimolecular recombination rate increases. The maximum amplification found at a very specific concentration demonstrates the importance of highly controlled mixing ratios and morphology achievable in vacuum-processed devices.

The dark current of the $1 \mathrm{wt} \%$ device is lower than that of the 2 wt $\%$ device, which we attribute to the smaller amount of D-A interfaces as well as to an increased number of traps ${ }^{45-47}$. However, comparing the dark current of $3 \mathrm{wt} \%$ and $4 \mathrm{wt} \%$ devices, we see that the former has a higher dark current and therefore a different behavior than the devices comprising $1 \mathrm{wt} \%$ and $2 \mathrm{wt} \%$. Analyzing the four devices together, we observe that an enhanced photocurrent, and thereby EQE, seems to be correlated with an increased dark current. Daanoune et al. suggested that this correlation is an intrinsic consequence of the working principle of devices based on enhanced injection by charge accumulation ${ }^{48}$. In the dark, charges are thermally activated over the bandgap of the system, which in a D-A heterojunction corresponds to energy of CT states. In an ideal diode, this current corresponds to the saturation current, $J_{0}^{46}$. In PM-OPDs, charge carriers forming $J_{0}$ accumulate in the same way that photo-generated carries do, leading to enhanced injection also of dark current. In the same study, the authors also correlate the rather slow speed of PM-OPDs to the slow trap dynamics. This not only explains the observed trend, but also indicates that this dark current effect might be detrimental for the specific detectivity $\left(D^{*}\right)$ of PM-OPD. This aspect is addressed in the following section.

The role of dark current in Photomultipliers. The specific detectivity $D^{*}$ of photodetectors depends on the EQE, which, as shown, can be enhanced by photomultiplication. However, it also depends on the noise of the device. We can express $D^{*}$ in terms of the device spectral noise density, $S_{\mathrm{n}}$, as follows:

$$
D^{*}=\frac{q \lambda}{h c} \frac{\mathrm{EQE}}{S_{\mathrm{n}}} .
$$

where $q$ is the elementary charge, $\lambda$ the wavelength, $h$ the Planck constant, and $c$ the speed of light. In the absence of a frequency dependent component of the noise, $S_{\mathrm{n}}$ reads:

$$
S_{\mathrm{n}}=\sqrt{2 q J_{\mathrm{D}}+\frac{4 k_{\mathrm{B}} T}{R_{\mathrm{sh}}}},
$$

which takes into account the shot and thermal noise, the first and second term in Eq. 2, respectively, where $J_{\mathrm{D}}$ is the dark current density, $k_{\mathrm{B}}$ the Boltzmann constant, $T$ the temperature and b

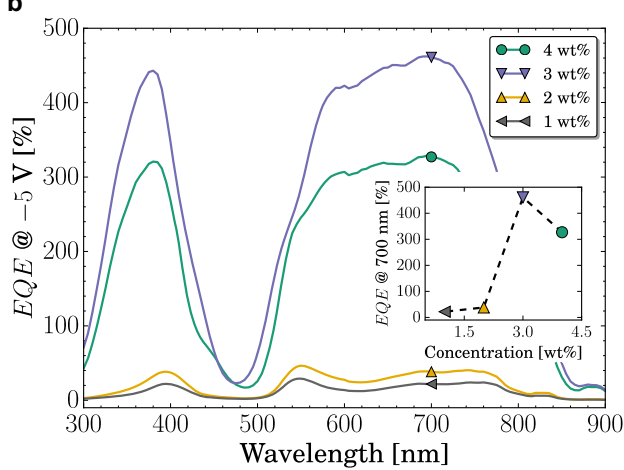

Fig. 2 Electrical characteristics of PM-OPD. a JV curves in dark (dashed lines) and under $100 \mathrm{~mW} \mathrm{~cm}^{-2}$ illumination (solid lines) of devices based on $\mathrm{ZnPc}: \mathrm{C}_{60}$ at different concentrations. A clear increase of the light and dark current is observed for devices with concentration higher than 3 wt\%. $\mathbf{b}$ EQE of the same devices showed in $\mathbf{a}$, measured at $-5 \mathrm{~V}$. The observed increase in current under illumination results in an increased $\mathrm{EQE}$ over $100 \%$, achieving maximum of $450 \%$. 

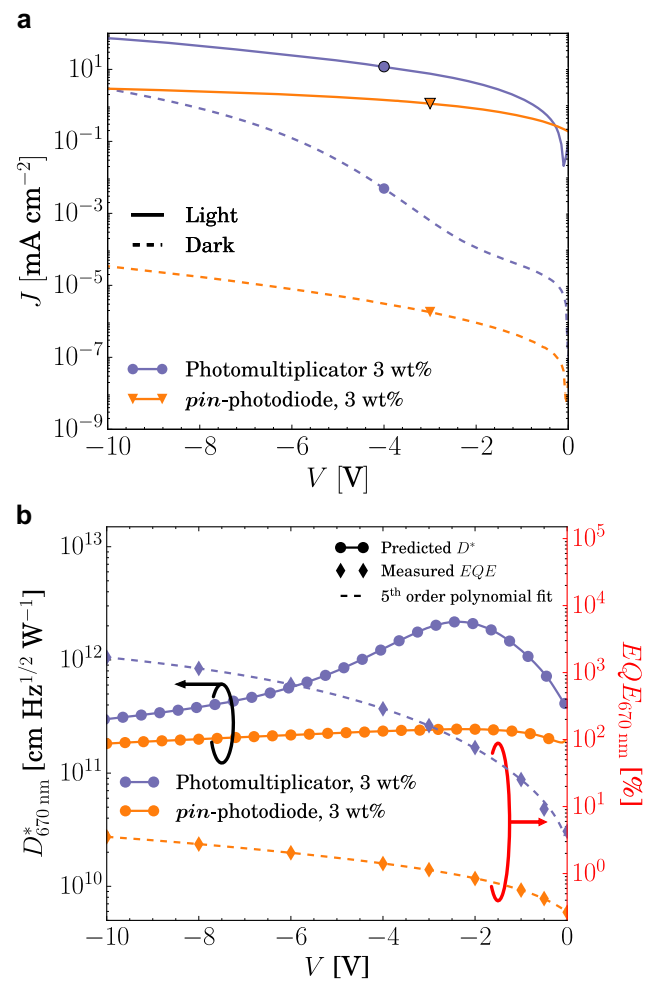

Fig. 3 Comparison of a PM-OPD and an equivalent pin-photodiode. a $\mathrm{JV}$ characteristics in dark and under $100 \mathrm{~mW} \mathrm{~cm}^{-2}$ illumination of a pinphotodiode and of a PM-OPD. $\mathbf{b}$ The EQE measured at different voltages is fitted with polynomial function from which the detectivity is predicted. The total noise is calculated from the shot and thermal noise, which are obtained from the dark current and the shunt resistance at room temperature, respectively. The PM-OPD shows a maximum $D^{\star}$ of $2.2 \times 10^{12}$ Jones around $-2.5 \mathrm{~V}$, which is higher than $D^{\star}$ of the equivalent pinphotodiode over the entire range measured, besides the high dark current at high reverse bias. The pin-photodiode comprises the same structure shown in Fig. 1c, except for the ETL, where instead of HATNA-Cl 6 , BPhen $(8 \mathrm{~nm})$ is used, see Supplementary Fig. 1b.

$R_{\mathrm{sh}}$ the shunt resistance normalized by the area $\left(\mathrm{m}^{2} \Omega\right)$ extracted from the inverse of the derivative of $J V$ curve around $0 \mathrm{~V}$. In most organic devices, however, the high reverse dark current makes the shot component the main source of noise, which represents a limitation also in diode-like organic photodetectors.

In polymer-based devices, different material systems have been reported to show high EQE; however, the values of the dark current have not always been presented. As mentioned before, the increase in photocurrent is usually correlated with an increased dark current. Therefore, both parameters have to be analyzed concomitantly in order to identify whether photomultiplication can be used to indeed get an increased $D^{*}$ as an equivalent pinphotodiode. In Fig. 3a, the dark current of the same devices shown in Fig. 2a is compared to that of a pin-photodiode comprising the same concentration. As can be seen, while the photocurrent reaches values two orders of magnitude higher than that of the pin-photodiode at $-10 \mathrm{~V}$, the dark current is four orders of magnitude higher, see Fig. 3a. Therefore, in order to overcome the performance of a pin-photodiode in terms of signal detectivity, EQE has to be as high as possible to even compensate such an increase in dark current. We have already shown that EQEs of almost $2000 \%$ can be achieved for small molecule devices. Now we must investigate if $D^{*}$ is indeed higher than that of equivalent pin-photodiodes.
In most well working pin-photodiodes, EQE is weakly dependent on applied bias, which can also be seen in Fig. 3a, where the photocurrent does not increase significantly with increasing reverse bias. Nonetheless, we measured the voltagedependent EQE (in Fig. 3b) and approximated its maximum value as function of voltage by a 5th order polynomial, which allows to have estimated values of EQE for every voltage. From the fit, together with the measured dark current and shunt resistance, the voltage-dependent $D^{*}$ can be calculated, according to Eq. (1) and Eq. (2). The same procedure is used for the PMOPD. The results are compared in Fig. $3 \mathrm{~b}$.

From Fig. 3 it is obvious that increasing EQE only is not sufficient to achieve high detectivities. As the dark current usually changes by orders of magnitude as a function of applied bias, the latter dominates $D^{*}$. Given this tradeoff, an optimum operation region has to be found, where the effect of the dark current does not overcome the enhancement in EQE. In the most favorable operation region, $D^{*}$ of $2.2 \times 10^{12}$ Jones is obtained for the photomultiplier device, comparable to results reported for PMOPDs and higher than $D^{*}$ provided by the equivalent pinphotodiode, where $D^{*}$ is in the order of $10^{11}$ Jones over the entire range measured. Whether the abrupt increase in dark current is indeed an intrinsic consequence of the photomultiplication process, as previously suggested, is still not clear. While it seems to be the case for most reported photomultipliers, some examples combine a high EQE with low dark currents, leading to high performance $^{17}$. If the dark current of the PM-OPDs would be comparable to the one obtained in the pin-photodiode, $D^{*}$ could be improved by two orders of magnitude. This shows that further investigations are needed to understand the origin of dark current in this device class.

Enhancement of charge-transfer state absorption/response in photomultipliers. We have shown that by controlling the D-A mixing ratio, photomultiplication can also be achieved in vacuum-processed organic blends in the spectral range of strong donor absorption. Whether the same effect is present when exciting in the CT absorption region is an important and, so far unaddressed question, which is relevant for microcavity CTOPDs. However, the low-acceptor concentration required for photomultiplication to take place decreases the number of D-A interfaces, establishing a tradeoff between enhanced $\mathrm{EQE}$ and absorption. In order to be useful, the amplified EQE in the CT absorption region should overcome the EQE of a standard photodiode-based device, in which a higher density of CT states is present.

In Fig. 3, we showed that the photomultiplier is superior the pin-photodiode based on the same blend ratio. However, $\mathrm{ZnPc}$ : $\mathrm{C}_{60}$ pin-photodiode was shown to produce maximum photocurrent when used at $50 \mathrm{wt} \%$ mixing ratio ${ }^{49}$. Therefore, in Fig. 4, the sensitively measured EQE spectra of a PM-OPD (3wt\%) at different bias are compared to that of a standard $\mathrm{ZnPc}: \mathrm{C}_{60}$ (50 wt $\%)$ pin-photodiode at zero bias. The CT band is observed for wavelengths longer than $800 \mathrm{~nm}$ in the EQE spectra of both devices. In the pin-photodiode, the CT band is more pronounced due to the higher density of CT states provided by the larger amount of D-A interfaces. In the same region, the PM-OPD shows a lower absorption shoulder, but also extending to the near-infrared region. As expected, at zero bias, the EQE of the PM-OPD is orders of magnitude lower than that of the pinphotodiode, as no enhanced injection takes place. By applying $-2 \mathrm{~V}$, however, the EQE in the visible range overcomes that of the pin-photodiode demonstrating the enhanced injection upon illumination. When $-5 \mathrm{~V}$ are applied to the device, the entire EQE spectrum of the PM-OPD surpasses that of the pin-photodiode, 
a

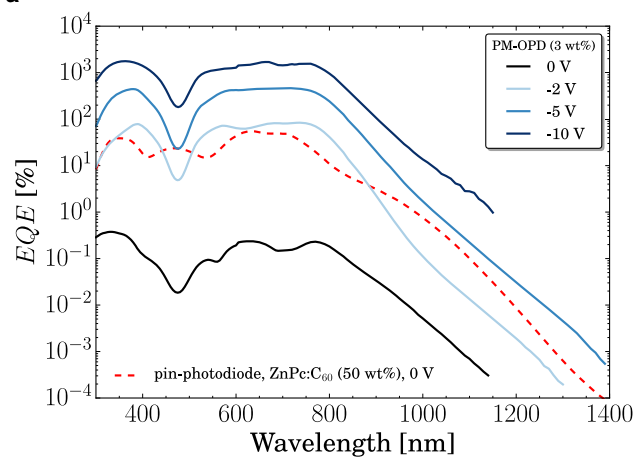

c

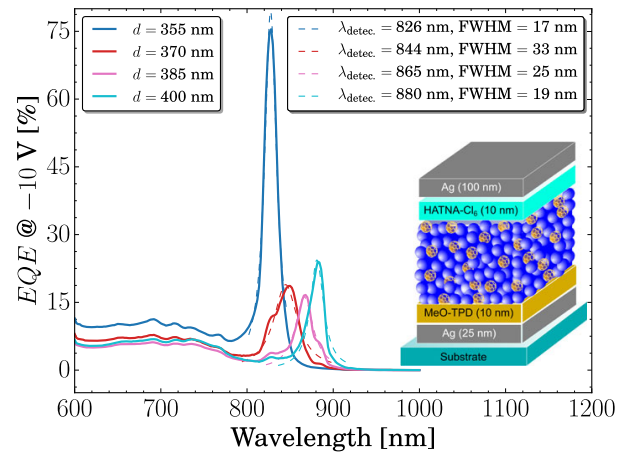

b
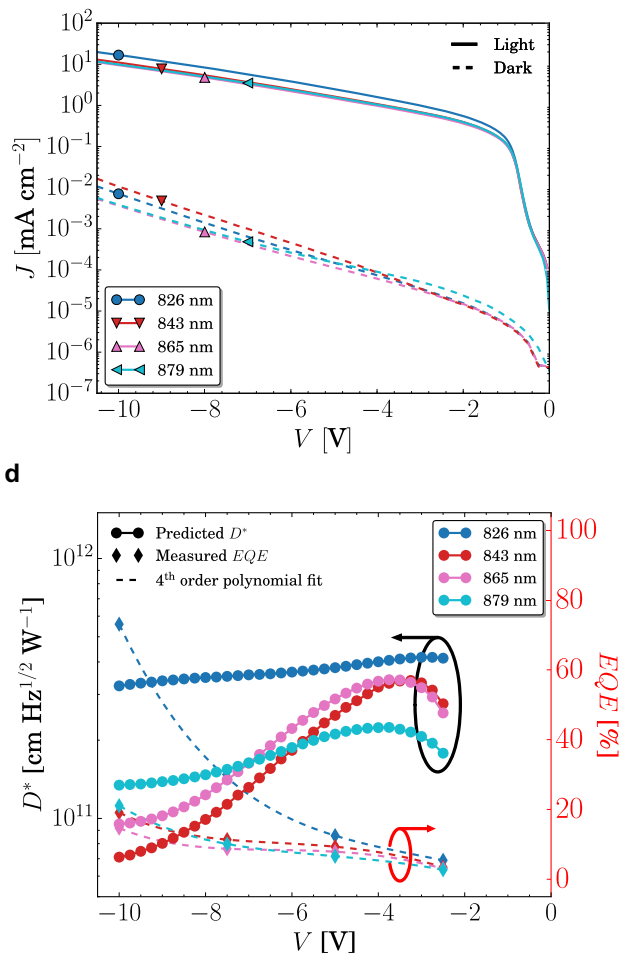

Fig. 4 Photomultiplication in the CT region and used in narrowband devices. a EQE as a function of excitation wavelength for the broadband photodetector. Red dashed line shows the EQE spectrum of a conventional $\mathrm{ZnPc}: \mathrm{C}_{60}(50 \mathrm{wt} \%$ ) photodiode and the black solid line the PM-OPD, both at $0 \mathrm{~V}$. Solid lines show the spectra of the PM-OPD $\left(3 \mathrm{wt} \% \mathrm{C}_{60}\right.$ ) under bias as indicated in the legend. Under $-5 \mathrm{~V}$, the higher injection provided by the energy level bending leads to an EQE higher that of a conventional pin-photodiode. $\mathbf{b} \mathrm{JV}$ characteristics under dark and under $100 \mathrm{~mW} \mathrm{~cm}^{-2}$ of four different narrowband devices with varying resonant wavelength as indicated in the legend. $\mathbf{c}$ EQE of cavities of the same devices shown in $\mathbf{b}$ at $-10 \mathrm{~V}$. As the active layer thickness increases from 355 to $400 \mathrm{~nm}$, the resonant wavelength redshifts from around 830 to $880 \mathrm{~nm}$. Dashed lines show the fit to a Lorentzian function, from which the FWHM is extracted. d EQE measured at different voltages is fitted with polynomial function, from which $D^{\star}$ is predicted. An optimum operation region is found around $-3.5 \mathrm{~V}$, where $D^{\star}$ of $6 \times 10^{11}$ Jones is obtained.

confirming that direct excitation of CT states can also trigger the photomultiplication process in these devices.

While the PM effect is commonly accompanied by an EQE above $100 \%$, it is the IQE which better defines the physical phenomenon behind this effect. In order to induce PM, free charge carriers must be firstly generated, requiring photons to be absorbed. As a means of quantifying whether absorbed photon induce enhanced injection in the sub-gap absorption region in our devices, we estimated their IQE, which accounts only for absorbed photons. To that end, we employ the transfer matrix method $^{50,51}$ (TMM) using ellipsometrically derived $n, k$-values to simulate the absorption in our devices and adjust the IQE to reproduce the magnitude of the measured EQE spectra, see Supplementary Fig. 6 for more details. Under $-10 \mathrm{~V}$ a constant IQE of $1750 \%$ over the full wavelength range, i.e., including CT absorption, is required to describe the experimental data as shown in Supplementary Fig. 1. Figure 4a together with TMM simulation data demonstrate the potential of combining such systems with optical cavities to accomplish high performance narrowband photodetectors.

In order to test whether such devices could be achieved, we embedded the best performing PM-OPD, i.e., $3 \mathrm{wt} \%$, into an optical microcavity, see inset in Fig. 4c for the device structure. Due to the higher Ag work function as compared to that of ITO, we inserted a $10 \mathrm{~nm}$-thick MeO-TPD layer to hinder hole injection in reverse bias. With aid of TMM, we simulate the optical photoresponse of a device comprising the same active layer thickness of $400 \mathrm{~nm}$, which leads to a resonant peak around
$880 \mathrm{~nm}$. Different resonant peaks can be achieved by varying the thickness of the active layer, leading to tunable near-infrared detection as shown in Supplementary Fig. 3. The $J V$ and EQE characteristics of devices comprising thicknesses from 355 to 400 $\mathrm{nm}$ are shown in Fig. 4b, c, respectively. As predicted by the optical simulation, narrowband peaks arise in the EQE spectra. As a demonstration, we tune the response wavelength from $\sim 830$ $\mathrm{nm}$ to $\sim 880 \mathrm{~nm}$, which under $-10 \mathrm{~V}$, reaches maximum EQE of $20-80 \%$, with a FWHM varying from 20 to $40 \mathrm{~nm}$. As to prove that photomultiplication also takes place in the narrowband devices, we estimate the IQE of these devices. Indeed, for the device with a detection wavelength of $828 \mathrm{~nm}$, an IQE of $160 \%$ is achieved. The three other devices show IQE of around $40 \%$, from which it is not possible to infer whether such values are a result of the PM effect or other phenomena. Therefore, to elucidate that, we increase the bias voltage to $-15 \mathrm{~V}$. This leads to EQEs and IQEs above $100 \%$ for all four devices, with peak values of $\sim 430 \%$ and $\sim 920 \%$, respectively, see Supplementary Fig. 4 .

Also in the microcavity devices, the dark current plays an important role in the final $D^{*}$. Although in these devices a better on/off ratio is kept along the reverse bias region as compared to those of Fig. 2, see Fig. 4b, the on/off ratio decreases as the reverse voltage increases, pointing to a decreased $D^{*}$ at high reverse bias. Therefore, we also estimate an optimum operation regime, where the tradeoff between EQE and dark current is maximized. As depicted in Fig. $4 \mathrm{~d}$, we obtain $D^{*}$ as high as $6 \times 10^{11}$ Jones in narrowband devices, which is comparable to narrowband pindevices based on cavities ${ }^{36,38}$. Moreover, it is superior than that of 
narrowband photomultiplication-type devices based on $\mathrm{CIN}^{41}$, where, in addition, excessively thick devices are demanded, which increases the operation voltage.

Transient photocurrent. Another important figure-of-merit of photodetectors is the response speed. In PM-OPDs, the temporal response is believed to be limited by the trapping/detrapping dynamics ${ }^{17,48}$, while other processes such as charge carrier transit time should be much shorter. In order to investigate the response speed of our devices, transient current measurements are performed. The rise time (from 10 to $90 \%$ of the device saturated signal) and fall time (from 90 to $10 \%$ of the device off signal) are summarized in Supplementary Table 1. The rise time of both broad- and narrowband devices ranges from 20 to $600 \mu \mathrm{s}$, corresponding to $-3 \mathrm{~dB}$ cutoff frequencies of $\sim 19.5$ to $\sim 0.4 \mathrm{kHz}$. These values are comparable to the best performing PM-OPDs reported so $\mathrm{far}^{7}$ and are suitable for health monitoring and videoframe-rate imaging applications.

\section{Discussion}

In CT-OPDs, the thicknesses required are much smaller than those used in narrowband devices based on charge collection narrowing $(\mathrm{CCN})^{52,53}$ or on $\mathrm{CIN}^{41,54}$. In the latter, for example, the thickness of the active layer must be much larger than the inverse of the absorption coefficient of the active layer, such that under illumination charges are generated close to the injecting contact, thereby causing the necessary band bending ${ }^{54}$. Moreover, in CT-OPDs the response can be further redshifted not only by increasing the thicknesses of the active layer, but also by introducing spacer/transport layers, which make the device electrically thin, but optically thick ${ }^{36-38}$. By combining the concept used in CT-OPDs with photomultiplication, we are able to achieve much thinner devices, as demonstrated in Fig. $4 \mathrm{~b}$-d, where active layers of $355 \mathrm{~nm}$ were used for spectral response at $\sim 830 \mathrm{~nm}$, compared to $2.5 \mu \mathrm{m}$ reported for spectral response at $650 \mathrm{~nm}$ when using CIN combined with photomultiplication ${ }^{41}$. The concept presented here can further benefit from the properties of microcavity devices while keeping enhanced $\mathrm{EQE}$ by photomultiplication at reasonable thicknesses and operation voltages. Moreover, in PMOPDs, the position of the active layer can be placed in an optimized position, either near the contact to enhance injection or such that optical overtones are minimized. There are systems combining low dark currents with enhanced $\mathrm{EQE}^{17}$, which, together with our concept, can potentially overcome the performance of state-of-art near-infrared narrowband devices.

In summary, we investigate the photomultiplication effect in fully vacuum-processed organic photodetecting devices. At $3 \mathrm{wt} \%$ of $\mathrm{C}_{60}$, a significant increase in $\mathrm{EQE}$ is observed under reverse bias, attributed to the accumulation of electrons caused by the lack of percolation paths. In the optimum operation regime, a specific detectivity $D^{*}$ of $\sim 10^{12}$ Jones is achieved. In addition, sensitively measured EQE spectra reveal that the enhancement extends to the CT absorption region, which indicates that these states also trigger photomultiplication, making microcavity CTOPDs with photomultiplication possible. Indeed, by exchanging top and bottom contacts by semitransparent mirrors, narrowband NIR PM-OPDs with response from 830 to $880 \mathrm{~nm}$ can be realized, achieving $D^{*}$ of $\sim 10^{11}$ Jones and FWHM as low as $20 \mathrm{~nm}$. The combination of optical microcavities with the photomultiplication effect can potentially boost NIR CT-OPDs, which so far were limited by the low $\mathrm{EQE}$ in the $\mathrm{CT}$ absorption region. Furthermore, much thinner devices are sufficient to achieve narrowband detection, as compared to the CIN approach. Additionally, the method presented here allows placing the active layer in different positions within the device or using gradients of
D-A mixing ratio, thereby enhancing injection and diminishing the effect of optical overtones, a critical problem in CT-OPDs.

\section{Methods}

Device preparation. Organic layers used in the devices were thermally evaporated on glass substrates covered by pre-structured ITO contact $\left(32 \Omega . \square^{-1}\right.$, Thin Film Devices) at ultrahigh vacuum (pressure $<10^{-7}$ mbar). Before deposition, substrates are cleaned for $15 \mathrm{~min}$ in different ultrasonic baths with NMP solvent, deionized water and ethanol followed by $\mathrm{O}_{2}$ plasma for $10 \mathrm{~min}$. Organic materials were purified 2-3 times via sublimation. The overlap of the bottom and top contact ( $\mathrm{Al}$ $100 \mathrm{~nm}$, Kurt J. Lesker) defines the device active area $\left(6.44 \mathrm{~mm}^{2}\right)$. After evaporation, samples are directly transferred to a glovebox with inert atmosphere, where they are encapsulated with a cover glass, fixed by UV hardened epoxy glue. A moisture getter (Dynic Ltd.) is inserted between top contact and the glass to hinder degradation.

Current-voltage characteristics. Illuminated $J V$ characteristics were measured using a source measurement unit (Keithley SMU 2400). The devices were illuminated at intensity of $100 \mathrm{~mW} \mathrm{~cm}^{-2}$ provided by a sun simulator (Solarlight Company Inc., USA). The intensity is calibrated by a Hamamatsu S1337 silicon photodiode. Dark JV characteristics were measured with a sensitive SMU (Keithley SMU 2635). Every measurement data point was acquired after steady-state conditions were achieved. The measurement is controlled by the software SweepMe! (https://sweep-me.net/).

External quantum efficiency (EQE). The current generated by the device under monochromatic light chopped at $170 \mathrm{~Hz}$ (Oriel Xe Arc-Lamp Apex Illuminator combined with Cornerstone $2601 / 4 \mathrm{~m}$ monochromator (Newport, USA)) is measured with a lock-in amplifier (Signal Recovery SR 7265$)$. A mask $\left(2.78 \mathrm{~mm}^{2}\right)$ is used to avoid edge effects. The same procedure is followed to monitor the light intensity, measured with a calibrated silicon photodiode (Hamamatsu S1337 calibrated by Fraunhofer ISE). EQE is obtained by the ratio of charge carriers generated by the device with the number of incoming photons.

Sensitive external quantum efficiency (sEQE). A chopped monochromatic light $(140 \mathrm{~Hz}$, quartz halogen lamp $(50 \mathrm{~W})$ used with a Newport Cornerstone 260 1/4 m monochromator) is shined to the device. The current generated at short-circuit conditions or at applied bias is fed into a current-voltage preamplifier (DHPCA100, FEMTO Messtechnik $\mathrm{GmbH}$ ) before being measured by a lock-in amplifier (Signal Recovery 7280 DSP). The time constant of the lock-in amplifier was chosen to be $500 \mathrm{~ms}$ and the amplification of the preamplifier was increased to resolve low photocurrents. Light intensity was obtained by using a calibrated silicon ( $\mathrm{Si}$ ) and indium-gallium-arsenide (InGaAs) photodiode.

Transient current measurements. To record current transients, the measured device was held at short-circuit, connected to the low impedance $(50 \Omega)$ input of an oscilloscope (DPO7354C, Tektronix). $100 \mathrm{~Hz}$ square waveform from a signal generator (Agilent 33600 A Series) was used to control a MOSFET (IRF630N) driving the high-power white LED (LED Engin, Osram Sylvania Inc.). The pulse length was set to $5 \mathrm{~ms}$, allowing device to reach a steady state before switching off the light. The signal from the device was pre-amplified (DHPCA-100, FEMTO Messtechnik $\mathrm{GmbH}$ ) prior to being recorded on the oscilloscope.

Ellipsometry. Variable-angle spectroscopic ellipsometry was performed with an M2000 UI (J.A. Woollam Co., Inc., Lincoln, USA, wavelength range: 245-1690 $\mathrm{nm}$ ). The uniaxial optical dispersion of a $100 \mathrm{~nm} \mathrm{ZnPc}$ layer doped with $3 \mathrm{wt} \% \mathrm{C}_{60}$ was obtained using an optical model $\left(\mathrm{Si} / \mathrm{SiO}_{2}(1 \mu \mathrm{m}) / \mathrm{ZnPc} \mathrm{C}_{60}(100 \mathrm{~nm}, 1\right.$ TaucLorentz and 5 Gaussian oscillators, energy positions coupled $z$ to $x y$ ) with sharp interfaces and additional EMA (50\% void, $50 \% \mathrm{ZnPc}_{60}$ ) roughness top-layer.

\section{Data availability}

All data generated in this study have been deposited in the Materials Cloud database under accession code https://doi.org/10.24435/materialscloud:8w-q6.

Received: 4 November 2020; Accepted: 22 June 2021; Published online: 15 July 2021

\section{References}

1. Thakur, R. Scanning LIDAR in Advanced Driver Assistance Systems and Beyond: building a road map for next-generation LIDAR technology. IEEE Consum. Electron. Mag. 5, 48-54 (2016). 
2. McCarthy, A. et al. Long-range time-of-flight scanning sensor based on highspeed time-correlated single-photon counting. Appl. Opt. 48, 6241-6251 (2009).

3. Rablau, C. Lidar: a new self-driving vehicle for introducing optics to broader engineering and non-engineering audiences. in 15th Conference on Education and Training in Optics and Photonics: ETOP 201911143 (Optical Society of America, 2019).

4. Chen, F.-C., Chien, S.-C. \& Cious, G.-L. Highly sensitive, low-voltage, organic photomultiple photodetectors exhibiting broadband response. Appl. Phys. Lett. 97, 103301 (2010).

5. Park, S. et al. Ultraflexible near-infrared organic photodetectors for conformal photoplethysmogram sensors. Adv. Mater. 30, 1802359 (2018).

6. Chow, P. C. Y. \& Someya, T. Organic photodetectors for next-generation wearable electronics. Adv. Mater. 32, 1902045 (2020).

7. Miao, J. \& Zhang, F. Recent progress on photomultiplication type organic photodetectors. Laser Photon. Rev. 13, 1800204 (2019).

8. Zhao, Z., Xu, C., Niu, L., Zhang, X. \& Zhang, F. Recent progress on broadband organic photodetectors and their applications. Laser Photon. Rev. 14, 2000262 (2020).

9. Hiramoto, M., Imahigashi, T. \& Yokoyama, M. Photocurrent multiplication in organic pigment films. Appl. Phys. Lett. 64, 187-189 (1994).

10. Matsunobu, G., Oishi, Y., Yokoyama, M. \& Hiramoto, M. High-speed multiplication-type photodetecting device using organic codeposited films. Appl. Phys. Lett. 81, 1321-1322 (2002).

11. Li, L. et al. Achieving EQE of $16,700 \%$ in P3HT:PC71BM based photodetectors by trap-assisted photomultiplication. Sci. Rep. 5, 9181 (2015).

12. Wang, W. et al. Highly sensitive polymer photodetectors with a broad spectral response range from UV light to the near infrared region. J. Mater. Chem. C. 3, 7386-7393 (2015).

13. Zhao, Z., Wang, J., Miao, J. \& Zhang, F. Photomultiplication type organic photodetectors with tunable spectral response range. Org. Electron. 69, 354-360 (2019).

14. Miao, J., Du, M., Fang, Y. \& Zhang, F. Acceptor-free photomultiplication-type organic photodetectors. Nanoscale 11, 16406-16413 (2019).

15. Hammond, W. T. \& Xue, J. Organic heterojunction photodiodes exhibiting low voltage, imaging-speed photocurrent gain. Appl. Phys. Lett. 97, 73302 (2010).

16. Guo, D., Yang, D., Zhao, J., Vadim, A. \& Ma, D. Role of interfaces in controlling charge accumulation and injection in the photodetection performance of photomultiplication-type organic photodetectors. J. Mater. Chem. C. 8, 9024-9031 (2020).

17. Guo, F. et al. A nanocomposite ultraviolet photodetector based on interfacial trap-controlled charge injection. Nat. Nanotechnol. 7, 798-802 (2012).

18. Fang, Y., Guo, F., Xiao, Z. \& Huang, J. Large gain, low noise nanocomposite ultraviolet photodetectors with a linear dynamic range of $120 \mathrm{~dB}$. Adv. Opt. Mater. 2, 348-353 (2014).

19. Zhou, X. et al. Ultrahigh gain polymer photodetectors with spectral response from UV to near-infrared using $\mathrm{ZnO}$ nanoparticles as anode interfacial layer. Adv. Funct. Mater. 26, 6619-6626 (2016).

20. Jang, M. S., Yoon, S., Sim, K. M., Cho, J. \& Chung, D. S. Spatial confinement of the optical sensitizer to realize a thin film organic photodetector with high detectivity and thermal stability. J. Phys. Chem. Lett. 9, 8-12 (2018).

21. Li, X., Li, X., Wang, S. \& Xiao, Y. A trap-assisted ultrasensitive near-infrared organic photomultiple photodetector based on Y-type titanylphthalocyanine nanoparticles. J. Mater. Chem. C. 4, 5584-5592 (2016).

22. Lee, J. W., Kim, D. Y. \& So, F. Unraveling the gain mechanism in high performance solution-processed PbS infrared PIN photodiodes. Adv. Funct. Mater. 25, 1233-1238 (2015).

23. Nie, R. et al. Highly sensitive and broadband organic photodetectors with fast speed gain and large linear dynamic range at low forward bias. Small 13, 1603260 (2017).

24. Wu, Y., Fukuda, K., Yokota, T. \& Someya, T. A highly responsive organic image sensor based on a two- terminal organic photodetector with photomultiplication. Adv. Mater. 31, 1903687 (2019).

25. Lan, Z. et al. Near-infrared and visible light dual-mode organic photodetectors. Sci. Adv. 6, 1-9 (2020)

26. Dong, R. et al. High-gain and low-driving-voltage photodetectors based on organolead triiodide perovskites. Adv. Mater. 27, 1912-1918 (2015).

27. Liu, C. et al. PbS quantum dots-induced trap-assisted charge injection in perovskite photodetectors. Nano Energy 30, 27-35 (2016).

28. Luo, X. et al. Insight into trap state dynamics for exploiting current multiplication in organic photodetectors. Phys. Status Solidi Rapid Res. Lett. 10, 485-492 (2016).

29. Yang, D. et al. Deep ultraviolet-to-NIR broad spectral response organic photodetectors with large gain. J. Mater. Chem. C. 4, 2160-2164 (2016).

30. Reynaert, J., Arkhipov, V. I., Heremans, P. \& Poortmans, J. Photomultiplication in disordered unipolar organic materials. Adv. Funct. Mater. 16, 784-790 (2006).
31. Huang, J. \& Yang, Y. Origin of photomultiplication in $C \$ \_\{60\} \$$ based devices. Appl. Phys. Lett. 91, 203505 (2007)

32. Guo, F., Xiao, Z. \& Huang, J. Fullerene photodetectors with a linear dynamic range of $90 \mathrm{~dB}$ enabled by a cross-linkable buffer layer. Adv. Opt. Mater. 1, 289-294 (2013).

33. Guo, D. et al. Structure design and performance of photomultiplication-type organic photodetectors based on an aggregation-induced emission material. Nanoscale 12, 2648-2656 (2020).

34. Lüssem, B., Riede, M. \& Leo, K. Doping of organic semiconductors. Phys. Status Solidi 210, 9-43 (2013).

35. Tietze, M. L. et al. Elementary steps in electrical doping of organic semiconductors. Nat. Commun. 9, 1-8 (2018).

36. Siegmund, B. et al. Organic narrowband near-infrared photodetectors based on intermolecular charge-transfer absorption. Nat. Commun. 8, 15421 (2017)

37. Tang, Z. et al. Polymer: fullerene bimolecular crystals for near-infrared spectroscopic photodetectors. Adv. Mater. 29, 1702184 (2017).

38. Kaiser, C. et al. Manipulating the charge transfer absorption for narrowband light detection in the near-infrared. Chem. Mater. 31, 9325-9330 (2019).

39. Goris, L. et al. Absorption phenomena in organic thin films for solar cell applications investigated by photothermal deflection spectroscopy. J. Mater. Sci. 40, 1413-1418 (2005).

40. Vandewal, K. et al. The relation between open-circuit voltage and the onset of photocurrent generation by charge-transfer absorption in polymer: Fullerene bulk heterojunction solar cells. Adv. Funct. Mater. 18, 2064-2070 (2008).

41. Wang, W. et al. Highly narrowband photomultiplication type organic photodetectors. Nano Lett. 17, 1995-2002 (2017).

42. Falkenberg, C., Leo, K. \& Riede, M. K. Improved photocurrent by using ndoped 2,3,8,9,14,15-hexachloro-5,6,11,12,17,18-hexaazatrinaphthylene as optical spacer layer in p-i-n type organic solar cells. J. Appl. Phys. 110, 124509 (2011).

43. Baranovskii, S. D. Theoretical description of charge transport in disordered organic semiconductors. Phys. Status Solidi Basic Res. 251, 487-525 (2014).

44. Zhang, M., Wang, H., Tian, H., Geng, Y. \& Tang, C. W. Bulk heterojunction photovoltaic cells with low donor concentration. Adv. Mater. 23, 4960-4964 (2011).

45. Wu, Z. et al. Noise and detectivity limits in organic shortwave infrared photodiodes with low disorder. npj Flex. Electron. 4, 1-6 (2020).

46. Vandewal, K., Tvingstedt, K., Gadisa, A., Inganäs, O. \& Manca, J. V. Relating the open-circuit voltage to interface molecular properties of donor:acceptor bulk heterojunction solar cells. Phys. Rev. B Condens. Matter Mater. Phys. 81, $1-8$ (2010).

47. Kublitski, J. et al. Reverse dark current in organic photodetectors and the major role of traps as source of noise. Nat. Commun. 12, 551 (2021).

48. Daanoune, M., Clerc, R., Flament, B. \& Hirsch, L. Physics of trap assisted photomultiplication in vertical organic photoresistors. J. Appl. Phys. 127, 055502 (2020).

49. Tietze, M. L. et al. Correlation of open-circuit voltage and energy levels in zinc-phthalocyanine: C60 bulk heterojunction solar cells with varied mixing ratio. Phys. Rev. B 88, 1-10 (2013).

50. Pettersson, L. A. A., Roman, L. S. \& Inganäs, O. Modeling photocurrent action spectra of photovoltaic devices based on organic thin films. J. Appl. Phys. 86, 487-496 (1999).

51. Kaiser, C., Zeiske, S., Meredith, P. \& Armin, A. Determining ultralow absorption coefficients of organic semiconductors from the sub-bandgap photovoltaic external quantum efficiency. Adv. Opt. Mater. 8, 1901542 (2020).

52. Armin, A., Jansen-van Vuuren, R. D., Kopidakis, N., Burn, P. L. \& Meredith P. Narrowband light detection via internal quantum efficiency manipulation of organic photodiodes. Nat. Commun. 6, 6343 (2015).

53. Yazmaciyan, A., Meredith, P. \& Armin, A. Cavity enhanced organic photodiodes with charge collection narrowing. Adv. Opt. Mater. 7, 1-8 (2019).

54. Wang, W. et al. Organic photodetectors with gain and broadband/ narrowband response under top/bottom illumination conditions. Adv. Opt. Mater. 6, 1800249 (2018).

55. Barlow, S. et al. Synthesis, ionisation potentials and electron affinities of hexaazatrinaphthylene derivatives. Chem. Eur. J. 13, 3537-3547 (2007).

56. Zhao, W. \& Kahn, A. Charge transfer at n-doped organic-organic heterojunctions. J. Appl. Phys. 105, 123711 (2009).

57. Schwarze, $M$. et al. Band structure engineering in organic semiconductors. Science 352, 1446-1449 (2016).

\section{Acknowledgements}

J.K. acknowledges the German Academic Exchange Service for the Ph.D. fellowship. J.B. acknowledges the DFG project VA 1035/5-1 (Photogen) and the Sächsische Aufbaubank through project no. 100325708 (InfraKart). E.B. thanks Roland Schulze (IPF) for performing the ellipsometry measurements. L.B. acknowledges the European Union's Horizon 2020 research and innovation program under Marie Skłodowska-Curie Grant Agreement number 722651 (SEPOMO). 


\section{Author contributions}

J.K., A.F., D.S., K.V., and K.L designed the experiments, prepared the detectors, and optimized the devices. J.K. and S.X. performed the standard characterization of detector and simulated the absorption spectra. L.B. measured the transient photocurrent and analyzed the data. E.B. analyzed the ellipsometry data and extracted the $n, k$-values used in the TMM simulations. J.K. and J.B. measured sensitive EQE spectra. A.F., D.S., and J.B., supervised their team members involved in the project, K.V. and K.L. supervised the overall project. All authors contributed to analysis and writing.

\section{Funding}

Open Access funding enabled and organized by Projekt DEAL.

\section{Competing interests}

A.F. is co-founder of "Axel Fischer und Felix Kaschura GbR", which provided the measurement software "SweepMe!”(sweep-me.net). The name of the program is mentioned in the experimental of the manuscript. The other authors declare that they have no conflict of interest.

\section{Additional information}

Supplementary information The online version contains supplementary material available at https://doi.org/10.1038/s41467-021-24500-2.

Correspondence and requests for materials should be addressed to J.K. or K.L.
Peer review information Nature Communications thanks Qiuming Yu and the other, anonymous, reviewer(s) for their contribution to the peer review of this work. Peer reviewer reports are available.

Reprints and permission information is available at http://www.nature.com/reprints

Publisher's note Springer Nature remains neutral with regard to jurisdictional claims in published maps and institutional affiliations.

(c) (i) Open Access This article is licensed under a Creative Commons Attribution 4.0 International License, which permits use, sharing, adaptation, distribution and reproduction in any medium or format, as long as you give appropriate credit to the original author(s) and the source, provide a link to the Creative Commons license, and indicate if changes were made. The images or other third party material in this article are included in the article's Creative Commons license, unless indicated otherwise in a credit line to the material. If material is not included in the article's Creative Commons license and your intended use is not permitted by statutory regulation or exceeds the permitted use, you will need to obtain permission directly from the copyright holder. To view a copy of this license, visit http://creativecommons.org/ licenses/by/4.0/.

(C) The Author(s) 2021 